\title{
A Tool for Patient-Focused Care Regarding Neoadjuvant Chemotherapy for Intrahepatic Cholangiocarcinoma
}

\author{
Katherine S. Cools, MD, MSCR ${ }^{1}$, and Evan S. Glazer, MD, PhD, FACS, FSSO ${ }^{1,2}$ \\ ${ }^{1}$ Department of Surgery, College of Medicine, The University of Tennessee Health Science Center, Memphis, TN; \\ ${ }^{2}$ Center for Cancer Research, The University of Tennessee Health Science Center, Memphis, TN
}

In this issue of Annals of Surgical Oncology, Utuama et al. use the National Cancer Database (NCDB) in a propensity score-matched model to investigate whether neoadjuvant chemotherapy impacts survival for patients with intrahepatic cholangiocarcinoma (ICC). ${ }^{1}$ Overall, only $8.4 \%$ of the cohort underwent neoadjuvant chemotherapy for resectable ICC. Utuama et al. found that for patients with stage II and III ICC, survival was improved with neoadjuvant chemotherapy compared with an upfront surgical approach (hazard ratio [HR] 0.58, 95\% confidence interval [CI] $0.37-0.91 ; p=0.02$ ). No survival advantage was seen when the stage I patients were included in both the unadjusted (HR 0.92, 95\% CI 0.64-1.31; $p=0.66$ ) and propensity score-matched analysis (HR 0.94, 95\% CI $0.62-1.40 ; p=0.72$ ). This study suggests there may be a significant role for the use of neoadjuvant therapy in stage II and III ICC patients to improve survival.

Currently, the National Comprehensive Cancer Network $(\mathrm{NCCN})$ guidelines for ICC recommend upfront surgery for all resectable, non-metastatic ICCs. The recommendation is follow this by adjuvant chemotherapy and chemoradiation in R1/R2 resections. ${ }^{2}$ Even for adjuvant therapy, there are limited randomized controlled trials evaluating the efficacy of this approach. ${ }^{3}$ Those trials that do exist have a heterogenous population of gallbladder cancer, extrahepatic cholangiocarcinoma, and ICC, making the data difficult to interpret since these cancers behave

(C) Society of Surgical Oncology 2021

First Received: 2 December 2020

Accepted: 3 December 2020;

Published Online: 8 January 2021

E. S. Glazer, MD, PhD, FACS, FSSO

e-mail: eglazer@uthsc.edu differently. Given the high recurrence rates, even with R0/ $\mathrm{R} 1$ resections, adjuvant chemotherapy is recommended in ICC.

Even less data exist for the use of neoadjuvant therapy in ICC. There are no published randomized clinical trials demonstrating the utility of neoadjuvant chemotherapy for patients with ICC, and while there have been many retrospective studies, the results have been mixed. Using the NCDB database, Yadav et al. compared neoadjuvant versus adjuvant chemotherapy in patients with cholangiocarcinoma (intra- and extrahepatic) and found an improved overall survival (OS; median OS 40.3 vs. 32.8 months; $p=0.01){ }^{4}$ however, this study included both intra- and extrahepatic cholangiocarcinoma and included all patients who received chemotherapy, either pre- or postoperatively. A different multi-institution cohort study looked at only ICC patients and found no difference in OS in patients who received preoperative chemotherapy compared with those who did not. ${ }^{5}$ This study was limited in that only a small proportion of patients received preoperative chemotherapy $(5.9 \%)$. Additionally, patients in the neoadjuvant therapy group had more advanced disease. ${ }^{5}$

The uncommon nature of ICC makes large, randomized controlled trials impractical; however, studies evaluating uncommon and aggressive cancers often use propensity scores in their analyses. In the current paper, Utama et al. use propensity score-matching to demonstrate improved survival for patients with stage II and III disease undergoing neoadjuvant therapy (HR $0.58,95 \%$ CI $0.37-0.91$; $p=0.02$ ). The individual propensity score is used to balance the differences in baseline variables between the treated and untreated groups, therefore reducing bias. ${ }^{6}$ In particular, propensity score matching is used when there are a limited number of treated patients (i.e. neoadjuvant utilization in ICC) compared with the control group (i.e. 
upfront surgery in ICC). In matching, control subjects are 'matched' with treated subjects on background covariates, i.e. age, year of diagnosis, comorbidities, and hospital type. ${ }^{6}$ This modeling helps to reduce the bias in non-randomized treatment assignment, which cannot be simply adjusted for by traditional methods (matching, stratifying, or covariant adjustment) due to the limited number of covariates for adjustment. ${ }^{6}$

In the face of a rare and aggressive cancer, new therapeutic options must be carefully considered and closely evaluated. Unfortunately, many details of the neoadjuvant regimens, timing, and completion are lost in the NCDB as a consequence of it being an administrative database. ${ }^{7}$ This work highlights the importance of a few clinical trials on the horizon. A new phase II clinical trial, set to begin in December 2020, will evaluate the effectiveness of neoadjuvant chemotherapy (oxaliplatin and gemcitabine) in patients with resectable ICC with a high risk of lymph node metastases (ClinicalTrials.gov identifier: NCT04523402). This trial may help answer the utility of neoadjuvant therapy in clinical stage III disease, but may not fully address stage II disease. Another multicenter, phase II trial is underway evaluating neoadjuvant combination immunotherapy and chemotherapy (toripalimab, gemcitabine, and oxaliplatin; ClinicalTrials.gov identifier: NCT04506281). We look forward to the results of these clinical trials.

We would like to commend Dr. Anaya and team on a thought-provoking manuscript regarding neoadjuvant chemotherapy for ICC. While there are still many questions to be answered, their data support a philosophical shift in our approach to ICC towards use of neoadjuvant chemotherapy in ICC patients in order to understand tumor biology, not necessarily to increase survival (although this approach may improve survival for some groups of paitents). In using this tool for proper patient selection, we theorize that we will enhance a patient-focused and patientcentered approach while we await more evidence from the aforementioned trials.

DISCLOSURES Katherine S. Cools and Evan S. Glazer have no real or apparent conflicts of interest to declare.

\section{REFERENCES}

1. Utuama O, Permuth J, Dagne G, et al. Neoadjuvant chemotherapy for intrahepatic cholangiocarcinoma: a propensity-score survival analysis supporting a selective approach. Ann Surg Oncol 2020:1-39.

2. National Comprehensive Cancer Network. Hepatobiliary Cancers (Version 5.2020). Available at: https://www.nccn.org/professiona 1s/physician_gls/pdf/hepatobiliary_blocks.pdf. Accessed 17 Nov 2020.

3. Primrose JN, Fox RP, Palmer DH, et al. Capecitabine compared with observation in resected biliary tract cancer (BILCAP): a randomised, controlled, multicentre, phase 3 study. Lancet Oncol. 2019;20(5):663-73. https://doi.org/10.1016/s1470-2045(18)30915 $-\mathrm{X}$

4. Yadav S, Xie H, Bin-Riaz I, et al. Neoadjuvant vs. adjuvant chemotherapy for cholangiocarcinoma: a propensity score matched analysis. Eur J Surg Oncol. Aug 2019;45(8):1432-38. h ttps://doi.org/10.1016/j.ejso.2019.03.023.

5. Buettner S, Koerkamp BG, Ejaz A, et al. The effect of preoperative chemotherapy treatment in surgically treated intrahepatic cholangiocarcinoma patients: a multi-institutional analysis. J Surg Oncol. 2017;115(3):312-18. https://doi.org/10.1002/jso.24524.

6. D'Agostino RB. Propensity score methods for bias reduction in the comparison of a treatment to a non-randomized control group. Stat Med. 1998;17(19):2265-81. https://doi.org/10.1002/(sici)1097-02 58(19981015)17:19\%3c2265::aid-sim918\%3e3.0.co;2-b.

7. Boffa DJ, Rosen JE, Mallin K, et al. Using the national cancer database for outcomes research: a review. JAMA Oncol. 2017;3(12):1722-28. https://doi.org/10.1001/jamaoncol.2016. 6905 .

Publisher's Note Springer Nature remains neutral with regard to jurisdictional claims in published maps and institutional affiliations. 